\title{
Neural Correlates of Induced Motion Perception in the Human Brain
}

\author{
Hiromasa Takemura, ${ }^{1,3}$ Hiroshi Ashida, ${ }^{4}$ Kaoru Amano, ${ }^{2,5}$ Akiyoshi Kitaoka, ${ }^{6,7}$ and Ikuya Murakami ${ }^{1}$ \\ ${ }^{1}$ Department of Life Sciences, University of Tokyo, Meguro-ku, Tokyo 153-8902, Japan, ${ }^{2}$ Department of Complexity Sciences and Engineering, University of \\ Tokyo, Kashiwa-shi, Chiba 277-8561, Japan, ${ }^{3}$ Japan Society for the Promotion of Science, Chiyoda-ku, Tokyo 102-8472, Japan, ${ }^{4}$ Graduate School of Letters, \\ Kyoto University, Sakyo-ku, Kyoto 606-8501, Japan, ${ }^{5}$ Precursory Research for Embryonic Science and Technology, Japan Science and Technology Agency, \\ Kawaguchi-shi, Saitama 332-0012, Japan, ${ }^{6}$ Core Research for Evolutional Science and Technology, Japan Science and Technology Agency, Chiyoda-ku, \\ Tokyo 102-0076, Japan, and 7Department of Psychology, Ritsumeikan University, Kita-ku, Kyoto 603-8577, Japan
}

A physically stationary stimulus surrounded by a moving stimulus appears to move in the opposite direction. There are similarities between the characteristics of this phenomenon of induced motion and surround suppression of directionally selective neurons in the brain. Here, functional magnetic resonance imaging was used to investigate the link between the subjective perception of induced motion and cortical activity. The visual stimuli consisted of a central drifting sinusoid surrounded by a moving random-dot pattern. The change in cortical activity in response to changes in speed and direction of the central stimulus was measured. The human cortical area hMT + showed the greatest activation when the central stimulus moved at a fast speed in the direction opposite to that of the surround. More importantly, the activity in this area was the lowest when the central stimulus moved in the same direction as the surround and at a speed such that the central stimulus appeared to be stationary. The results indicate that the activity in hMT + is related to perceived speed modulated by induced motion rather than to physical speed or a kinetic boundary. Early visual areas (V1, V2, V3, and V3A) showed a similar pattern; however, the relationship to perceived speed was not as clear as that in hMT + . These results suggest that hMT + may be a neural correlate of induced motion perception and play an important role in contrasting motion signals in relation to their surrounding context and adaptively modulating our motion perception depending on the spatial context.

\section{Introduction}

Visual motion perception does not simply depend on point-wise signals on the retina but relies on active signal interactions across adjacent retinal locations. The processing of spatial interactions of motion signal has at least two aspects, because brightness processing involves both identification of luminance-defined edges and enhancement of simultaneous brightness contrast between adjacent regions. The first aspect is detecting the existence of velocity differences between abutting regions to identify kinetic boundaries (Baker and Braddick, 1982; Regan, 1989). Neurophysiological studies have demonstrated that the primary visual cortex (V1) exhibits greater activation when visual stimuli include boundaries defined by relative motion, suggesting in-

Received Feb. 5, 2012; revised Aug. 2, 2012; accepted Aug. 9, 2012.

Author contributions: H.T., H.A., A.K., and I.M. designed research; H.T., H.A., A.K., and I.M. performed research; K.A. contributed unpublished reagents/analytic tools; H.T., H.A., and K.A. analyzed data; H.T., H.A., K.A., A.K., and I.M. wrote the paper.

This study was supported by the Nissan Science Foundation, Japan Society for the Promotion of Science (JSPS) Funding Program for Next Generation World-Leading Researchers Grant LZ004, JSPS Grant-in-Aid for Scientific Research Grant A22243044, and JSPS Grant-in-Aid for JSPS Fellows. We thank Masahiko Terao and Rumi Hisakata for supporting the eye movement recording. We also thank Hiroshi Horiguchi, Kendrick N. Kay, Lee Michael Perry, and Brian A. Wandell for supporting the population receptive field analysis.

The authors declare no competing financial interests.

This article is freely available online through the J Neurosci Open Choice option.

Correspondence should be addressed to Hiromasa Takemura at his present address: Department of Psychology, Stanford University, 450 Serra Mall, Stanford, CA 94305. E-mail: htakemur@stanford.edu.

DOI:10.1523/JNEUROSCI.0570-12.2012

Copyright $\odot 2012$ the authors $\quad 0270-6474 / 12 / 3214344-11 \$ 15.00 / 0$ volvement of early visual areas (Lamme et al., 1993; Reppas et al., 1997).

The second aspect is emphasizing the difference in motion signals, each of which is pooled over a relatively large region of the visual field. In a manner phenomenally analogous to simultaneous brightness contrast in which the same gray appears brighter in a black surround and darker in a white surround, motion contrast can evoke a vivid illusory motion called induced motion, such that a physically stationary stimulus appears to move in the direction opposite to surrounding motion, and the perceived speed of a central stimulus that is itself moving in one direction becomes faster when a surround moves in the opposite direction (Duncker, 1929; Walker and Powell, 1974; Tynan and Sekuler, 1975; Reinhardt-Rutland, 1988). Because the spatial properties of induced motion are at least superficially consistent with the direction-dependent surround suppression in the macaque middle temporal area (MT) and medial superior temporal area (MST) neurons (Allman et al., 1985; Tanaka et al., 1986; Eifuku and Wurtz, 1998), it has been argued that these areas constitute a neural mechanism mediating induced motion (Murakami and Shimojo, 1993, 1996; Tadin et al., 2003). However, no evidence for this relationship is currently available because the relationship between the perception of induced motion and neural activities in the same species has never been examined.

Neuroimaging techniques, such as functional magnetic resonance imaging (fMRI), make it possible to examine this relationship by allowing a direct comparison between subjective reports 
A
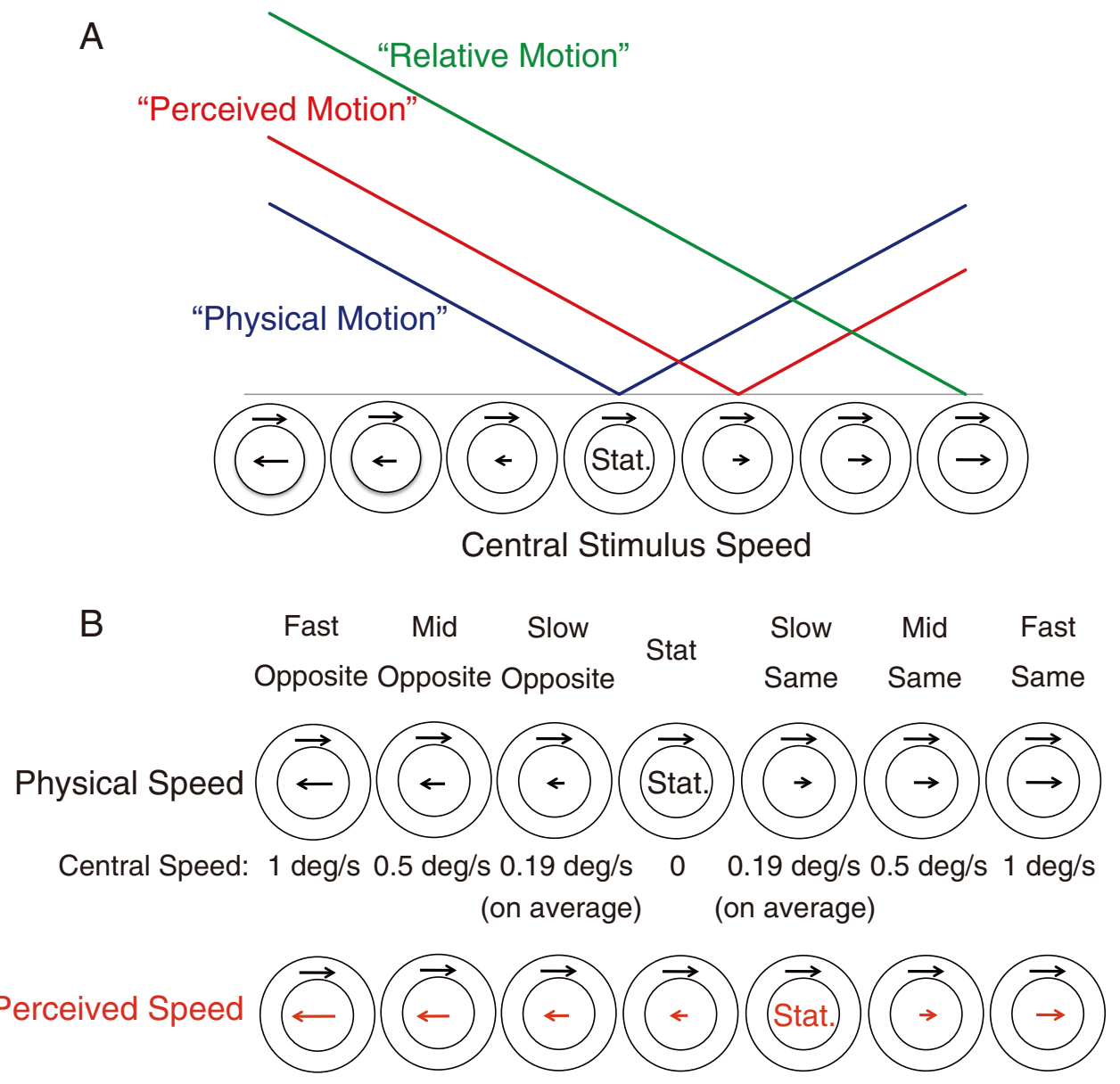

Figure 1. A, Schematic illustration of the three hypotheses. Horizontal axis schematizes the speed and direction of the central stimulus (note that the speed of the surround stimulus is constant). Vertical axis depicts the hypothetical amplitude of neural activation. Each colored line indicates the predicted neural activation pattern when the activity is dependent on physical speed (blue), perceived speed modulated by induced motion (red), and the difference in speed between the center and surround (green). Note that the linearity of speed dependence is assumed here only for illustrative purposes but is not specifically tested; the critical point is the locations of the minimum activation, which the three hypotheses predict differently. $\boldsymbol{B}$, Seven stimulus velocities. The velocity of the surrounding stimulus was identical across all conditions (1\%). The central stimulus speed is shown in the row labeled Central Speed.

and brain activity for the same stimulus in the same subject. Neuroimaging studies of motion-processing cortical areas (Shulman et al., 1998; Moutsiana et al., 2011) have demonstrated that opposing motions activate the human MT complex (hMT + ), the putative human homolog of the macaque MT and MST, to a greater extent than does unidirectional motion. However, these studies did not clarify whether this was attributable to kinetic boundaries characterized by a speed difference, to the patterns of complex optic flow fields, or to the occurrence of spatial interactions that are commonly used to calculate object velocities in moving contexts and to produce induced motion.

Using fMRI, the present study aimed to clarify the neural correlates of induced motion by dissociating them from the neural representations of physical motion and kinetic boundaries. Figure $1 \mathrm{~A}$ depicts our idea. We systematically manipulated the velocity of a central stimulus and examined whether cortical activation changed depending on physical speed, perceived speed, or relative speed.

\section{Materials and Methods}

\section{Subjects}

Nine healthy adults (three females; mean age, 26.7 years) participated in the fMRI experiment. All provided written informed consent. All experiments were approved by the Safety Committee of the Brain Activity Imaging Center of the Advanced Telecommunications Research Institute
International (ATR-BAIC, Kyoto, Japan) and the Ethics Committee of Ritsumeikan University (Kyoto, Japan). The experiments were conducted in accordance with the Declaration of Helsinki.

\section{Stimulus presentation}

Subjects viewed visual stimuli projected on a screen in the MRI bore through an oblique mirror mounted on the head coil. The stimulus image was generated by a personal computer and rear projected using a data projector (DLA-G150CL; Victor). All of the stimuli were generated using the MATLAB programming environment (MathWorks) and the Psychophysics Toolbox routines (Brainard, 1997). The spatial resolution was $1024 \times 768$ pixels, and the refresh rate was 60 frames/s. The distance from the eye to the screen was $96 \mathrm{~cm}$, and the screen size was $33.7 \times 25.4$ $\mathrm{cm}\left(19.3 \times 14.8^{\circ}\right.$ in visual angle). Those subjects who used glasses wore plastic correction lenses in the scanner.

\section{Stimuli and procedure}

Figure $2 \mathrm{~A}$ shows a screenshot of the stimuli we used. Six stimulus patches were presented around a fixation point (eccentricity at the center of each patch, $5.33^{\circ}$ ) and moved identically. Each patch was composed of central and surrounding stimuli. Each central stimulus was a Gabor patch (i.e., a drifting sinusoidal luminance modulation windowed by a Gaussian contrast envelope) with a sinusoid spatial frequency of $1 \mathrm{cycle} /{ }^{\circ}$, the envelope with a $0.62^{\circ} \mathrm{SD}$, and a Michelson contrast of $99 \%$. Each surrounding stimulus was a random-dot pattern of luminance modulation filtered using a bandpass spatial-frequency filter (center frequency, 1 cycle $/^{\circ}$ ). The inner and outer diameters of the surrounding stimulus were $1.96^{\circ}$ 


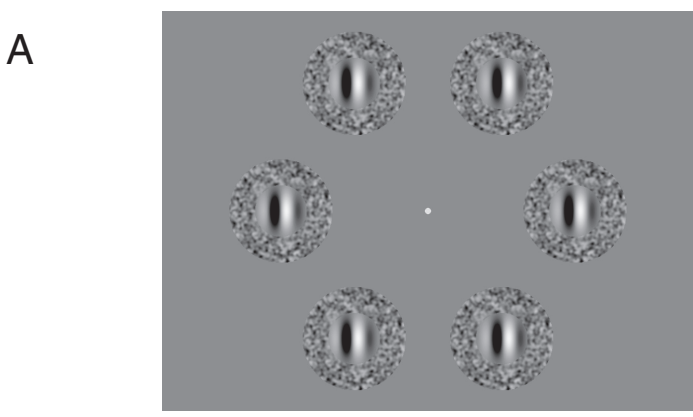

\section{$\mathrm{B}$}
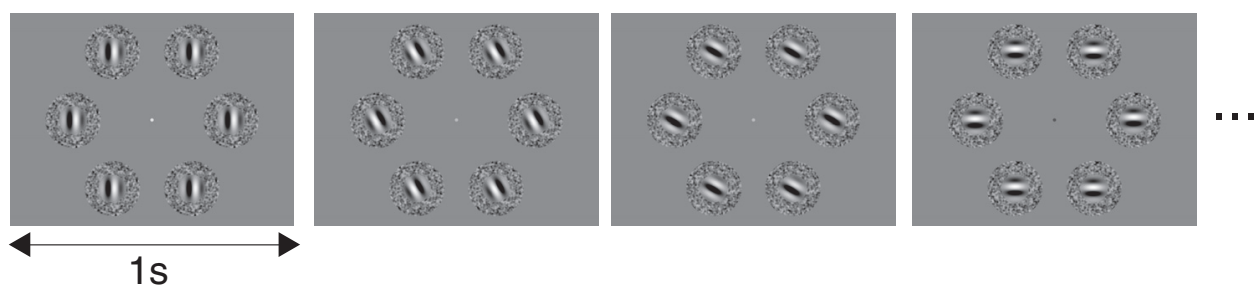

Figure 2. Visual stimuli. $\boldsymbol{A}$, The stimuli consisted of six Gabor patches, each surrounded by a random-dot kinematogram. $\boldsymbol{B}$, An example of the stimulus presentation sequence used in a typical block. The orientation and direction of the central and surrounding stimuli were abruptly changed by $30^{\circ}$ every second while the relationship of motion directions between the central and surrounding stimuli was kept constant.

and $3.92^{\circ}$, respectively. The speed of the surrounding stimulus was $1 \%$ throughout all conditions. The speed and direction of the central stimulus was varied across conditions, as described below.

The visual stimuli were presented in a block sequence. Each stimulus block of $15 \mathrm{~s}$ duration was followed by a uniform gray screen ("rest") of $15 \mathrm{~s}$ duration. This sequence was repeated seven times within each run. Before starting the stimulus presentation, we presented the rest screen for $15 \mathrm{~s}$. The fMRI images taken during this period were discarded before statistical analysis. The total length of each run was 225 s. All subjects were tested with seven such runs.

Figure $2 B$ shows a set of schematic examples of the motion directions used in a typical block. Within each $15 \mathrm{~s}$ stimulus block, the orientation and direction of the central and surrounding stimuli were rotated counterclockwise by $30^{\circ}$ every second while the relationship in terms of motion direction between the central and surrounding stimuli was kept constant. This overall rotation was introduced to stimulate a large population of neurons tuned to various directions and orientations and to avoid an oblique effect (Furmanski and Engel, 2000).

\section{Determination of cancellation velocity}

Before each fMRI experiment, the magnitude of induced motion was psychophysically determined for each subject. In the first step, each subject was seated outside the scanner and was presented with the stimulus moving in a horizontal or vertical direction for $1 \mathrm{~s}$ in each trial. The central stimulus was moved in the same or opposite direction relative to the surrounding stimulus, which always moved at $1 \%$. The central stimulus moved at $0,0.03,0.1,0.32$, or $1 \%$ s. Two blocked sessions were performed. In one block, both the central and surrounding stimuli moved in a vertical direction (upward or downward). In the second block, both stimuli moved in a horizontal direction (right or left). The stimulus size, position, and eccentricity were identical to those used in the fMRI experiment. After the stimulus presentation, the subject was asked to judge the motion direction of the central stimulus (either "upward" or "downward" in the vertical block; either "left" or "right" in the horizontal block). The cancellation velocity, or the velocity of the central stimulus at which it appeared stationary, was determined using the method of constant stimuli and by fitting a logistic psychometric function to the data using the maximum-likelihood method (Wichmann and Hill, 2001a). Figure $3 A$ shows examples of psychometric functions for a representative subject. The slope $\pm \mathrm{SD}$ of the function at the cancellation velocity was $2.06 \pm 1.121$ on average. We determined the cancellation velocity for each of the psychometric functions for the vertical and horizontal blocks. The $95 \%$ bootstrap confidence interval (Wichmann and Hill, 2001b) of the cancellation velocity overlapped with $0 \%$ only in one of the two blocks for two subjects and in neither block for the others. We used the average of the cancellation velocities between the vertical and horizontal blocks as the tentative cancellation velocity in the second step. In the second step, each subject was psychophysically tested inside the scanner to validate the cancellation velocity. The subject observed the stimuli in the same sequence as that subsequently used in the stimulus blocks in the actual fMRI sessions. The subject was asked to report whether the central stimulus appeared to move with the surround, to move opposite to the surround, or to be stationary based on the overall impression in the $15 \mathrm{~s}$ interval during which the motion direction was changed every second (see above, Stimuli and procedure). The central stimulus in the first trial moved at the tentative cancellation velocity that had been predetermined in the first step. Four of the subjects reported that the central stimulus was perceived as stationary in the first trial. For these subjects, we used this initially determined cancellation velocity in the main fMRI experiment and did not run subsequent trials. The remaining five subjects reported that the central stimulus appeared to be moving. For these subjects, it was possible that the cancellation velocity determined outside the scanner was slightly suboptimal. Therefore, the central stimulus speed was varied in small steps to search for the true cancellation velocity in the scanner environment. Figure $3 B$ shows an example. Typically, subjects reported the central stimulus as stationary within a certain range of speeds. This validation step ended when subjects reported that the central stimulus was perceived as moving again. In these cases, the average of the speeds at which the central stimulus was reported as stationary was used as the cancellation velocity in the main fMRI experiment. The average change of cancellation velocity in the second step was $0.06 \pm 0.09^{\circ} \%$ (mean $\pm \mathrm{SD}$ ).

The resulting cancellation velocity was $0.19 \pm 0.06 \%$ (mean $\pm \mathrm{SD}$ ); hence, sufficient induced motion was elicited inside the scanner.

\section{Experimental conditions and procedure}

Seven central stimulus velocities were used in the experiment (Fig. $1 B$ ). Under the "Fast Opposite" and "Mid Opposite" conditions, the central stimulus velocity was opposite to the surround and moved at 1 and $0.5 \%$, respectively. Under the "Slow Opposite" condition, the movement was opposite to the surround and at the absolute speed of the cancellation velocity, which was determined for each subject. Under the "Stat" con- 
A

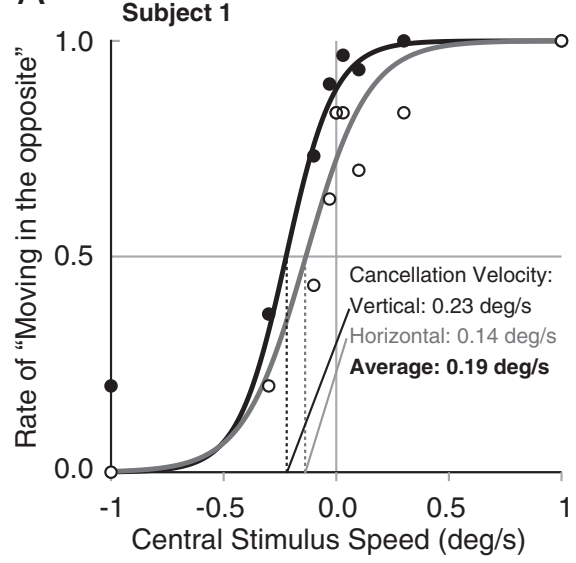

Subject 7

\begin{tabular}{lll} 
& Central speed & \multicolumn{1}{c}{ Subjective Report } \\
\hline Trial 1 & $0.2 \mathrm{deg} / \mathrm{s}$ & "Opposite direction" \\
Trial 2 & $0.216 \mathrm{deg} / \mathrm{s}$ & "Stationary" \\
Trial 3 & $0.233 \mathrm{deg} / \mathrm{s}$ & "Stationary" \\
Trial 4 & $0.25 \mathrm{deg} / \mathrm{s}$ & "Stationary" \\
Trial 5 & $0.268 \mathrm{deg} / \mathrm{s}$ & "Same direction"
\end{tabular}

Cancellation Velocity $=0.233 \mathrm{deg} / \mathrm{s}$

Figure 3. Determining cancellation velocity. $A$, Examples of psychometric functions for a representative subject obtained from a psychophysical experiment outside the scanner. The black and white circles indicate the results of the vertical and horizontal blocks, respectively. The results from each block were fitted with a logistic function. $\boldsymbol{B}$, Example of cancellation velocity validation inside the scanner. The speed of the central stimulus was varied in small steps. In the main fMRI experiment, we used the average of the central speeds within the range in which subjects reported the central stimulus as being stationary.

dition, the central stimulus was stationary. Under the "Slow Same" condition, the central stimulus moved in the same direction as the surround and at the cancellation velocity. Thus, this was the sole condition under which the central stimulus appear stationary to each subject. Under the "Mid Same" and "Fast Same" conditions, the central stimulus moved in the same direction as the surround and at 0.5 and $1 \%$, respectively. In each fMRI run, these seven velocities appeared in random order.

\section{Attention task}

BOLD signal changes may be affected by the state of attention (Huk et al., 2001); thus, we introduced an attention task used by Kuriki et al. (2008) to control attention. Every $0.5 \mathrm{~s}$, the color of the fixation point changed to one of five alternatives (red, yellow, green, blue, and purple) in random order. Each subject was instructed to fixate on the fixation point when it was displayed and to count the number of times the blue fixation point occurred during each run. The blue point appeared 84.3 times per run, on average. Thus, the task consisted of monitoring the fixation point, detecting each occurrence of the blue fixation point, and maintaining/ updating the number of occurrences in the working memory. This was a highly attention-demanding task, but all participants were able to perform it with an accuracy rate of $>95 \%$, indicating less than five misses per 100 occurrences.

\section{Region of interest localizing experiments}

$h M T+$ localizer. The location and size of hMT+ (Zeki et al., 1991; Watson et al., 1993; Tootell et al., 1995) were determined by the functional responses to stimuli that alternated between moving and stationary dot patterns. Previous studies have shown that this type of localizer stimulates both hMT and hMST, the putative human homologs of the macaque areas MT and MST (Huk et al., 2002; Wall et al., 2008; Amano et al., 2009b), but not the self-motion-related areas, such as V6 (Pitzalis et al., 2010). In this hMT + localizer, a $12 \mathrm{~s}$ motion block and a $12 \mathrm{~s}$ stationary block were paired. During the motion block, 200 white dots on a black background were presented within a circular aperture $\left(20^{\circ}\right.$ diameter) centered at the fixation point. The dots $\left(0.25^{\circ}\right.$ wide $)$ moved toward and away from the fixation point at $8 \%$, alternating directions every second. Each dot lasted for $167 \mathrm{~ms}$ (10 frames), after which it was replaced by another dot at a randomly selected position. The pair of motion/stationary blocks was repeated 13 times in each fMRI run, which lasted for $5.4 \mathrm{~min}$. The $12 \mathrm{~s}$ interval at the beginning of each fMRI run allowed the hemodynamic response to reach a stable baseline. We took the BOLD contrast between motion and stationary blocks and defined each hMT + region by identifying voxels that showed statistically significant BOLD changes at the significance level of $q<0.05$ using the falsediscovery rate (FDR)-controlling procedure.
Visual field mapping and population receptive field analysis. The boundaries between the retinotopic areas, V1, V2, V3, and V3A (V3A could not be identified in one of our subjects), and a boundary between two visual maps within hMT+ (temporal occipital areas TO-1 and TO-2; Amano et al., 2009b) were identified using the standard visual field mapping procedure. This procedure, which used a rotating wedge and an expanding ring (Sereno et al., 1994, 1995; Engel et al., 1997), has been reported to accurately detect the visual field maps of areas with a large population of receptive fields such as that of hMT + (Dumoulin and Wandell, 2008; Amano et al., 2009b). We also used moving bar stimuli to estimate the sizes of population receptive fields (pRFs; i.e., the region of visual space that stimulates the voxel of interest) within hMT + (Dumoulin and Wandell, 2008; Amano et al., 2009b). A dartboard pattern was exposed by slowly moving an aperture in the shape of a rotating wedge, an expanding ring, and a moving bar. Within the aperture, the pattern moved at $2 \mathrm{~Hz}$, with its motion direction changed randomly every $2-3$ s. The aperture positions were displaced in discrete steps in synchrony with the timing of each fMRI volume acquisition. The wedge aperture subtended $45^{\circ}$, and the width of the ring and bar was one-third of the stimulus radius. The $12 \mathrm{~s}$ interval at the beginning of each fMRI run allowed the hemodynamic parameters to reach a stable baseline. A full cycle of the wedge and ring stimuli took $24 \mathrm{~s}$, with a total of 6 cycles (144 s) per fMRI run. In total, each run lasted $156 \mathrm{~s}$. Four bar orientations $\left(0^{\circ}\right.$, $45^{\circ}, 90^{\circ}$, and $135^{\circ}$ from vertical) and two different motion directions orthogonal to each bar orientation were used, giving a total of eight different bar configurations within a given $192 \mathrm{~s} \mathrm{scan}$. Four runs (wedge/ ring) and six runs (bar) were performed for each subject.

Retinotopic maps were created by projecting the temporal phase delay of the response onto segmented and flattened cortical surfaces. The borders between visual areas were marked manually at the reversals between phase-map colors.

We used a model-based method to estimate pRFs to validate the distinction among visual field maps within hMT + . We predicted the BOLD response of each voxel using a two-dimensional Gaussian pRF model with a center location $(x, y)$ and spread $(\sigma)$ as parameters. The predicted fMRI time series was calculated by a convolution of the model pRF with the stimulus sequence and two-gamma hemodynamic response function (HRF; Friston et al., 1998; Glover, 1999; Worsley et al., 2002). The pRF parameters for each voxel were determined by minimizing the sum of the squared residuals between the predicted and observed fMRI time series for all stimuli (wedges, rings, and bars). We excluded voxels with poor pRF model fits from the analysis (variance explained $<30 \%$ ). See previous studies for additional details of the pRF analysis (Dumoulin and Wandell, 2008; Harvey and Dumoulin, 2011).

Stimulus localizer. We used a stimulus localizer to extract the voxels that responded to the central stimulus of each of the six patches. A $12 \mathrm{~s}$ stimulus block and a $12 \mathrm{~s}$ rest block were paired in the stimulus localizer run. In the stimulus block, dynamic random noise was presented in the display regions corresponding to the locations of the central stimuli. Each pixel of the dynamic random noise had one of two luminance values - black or white-with a probability of $50 \%$ for each, and it was refreshed every two frames. In the rest block, only the fixation point was presented on a uniform gray screen. The stimulus/ rest pair was repeated three times in each fMRI run. We selected voxels that showed BOLD changes between the stimulus and rest blocks (uncorrected $p<0.05$ ). The threshold had to be lowered to extract voxels in extrastriate areas such as hMT + in which a smaller signal-to-noise ratio is available (Wandell and Winawer, 2011). We also used stricter cutoffs (uncorrected $p<0.005$; FDR-corrected $q<$ $0.05)$ to confirm the robustness of the data. 


\section{MRI data acquisition and analyses}

We used a 3 T MRI scanner (Magnetom Verio; Siemens) equipped at ATR-BAIC with a 12-channel head coil. An anatomical image of the whole brain was taken using the T1-weighted protocol (MPRAGE sequence; TR, $2250 \mathrm{~ms}$; TE, $3.1 \mathrm{~ms}$; flip angle, $9^{\circ}$ ) at a spatial resolution of $1 \times 1 \times 1 \mathrm{~mm}^{3}$. Region of interest (ROI) analyses were made after the alignment of each functional image to the anatomical image. All functional images were taken under identical parameters using the EPI technique with the $\mathrm{T} 2{ }^{\star}$-weighted protocol (field echo-EPI sequence; TR, $3000 \mathrm{~ms}$; TE, $40 \mathrm{~ms}$; flip angle, $\left.80^{\circ}\right)$. The in-plane resolution was $2 \times 2$ $\mathrm{mm}^{2}\left(\mathrm{FOV}, 200 \times 200 \mathrm{~mm}^{2}\right.$ at $100 \times 100$ pixel $^{2}$ ), and 31 slices, each 2 $\mathrm{mm}$ thick, were taken in near-axial planes that were parallel to the anterior commissural-posterior commissural line so that the lateral occipital and temporal occipital cortices were covered.

We used BrainVoyager QX software (Brain Innovation) to process and analyze the MRI images. For preprocessing, we applied slice-timing correction, motion correction, and temporal high-pass filtering (cutoff, 3 cycles/run). The ROI analysis was applied following the co-registration process. We used each individual subject's head coordinates rather than normalized coordinates because the location of hMT + differs across subjects (Dumoulin et al., 2000). We also used custom software (mrVista software package for MATLAB, which is freely available at http://vistalab. stanford.edu/software/) to estimate the pRF sizes within hMT + (Dumoulin and Wandell, 2008; Amano et al., 2009b).

We defined the baseline BOLD signal as the average across the three scans taken before the stimulus onset in the data analysis $(-6,-3$, and $0 \mathrm{~s}$ ). We defined the response amplitude by averaging the signal change values of four scans $(6,9,12$, and $15 \mathrm{~s}$ after stimulus onset) around the peak of the curve showing activation in response to the stimulus presentation. To examine the robustness of the results, we also redefined the amplitude of the responses using model responses (the stimulus time course convolved with the HRF). We used the default two-gamma HRF from the SPM5 package (http://www.fil.ion.ucl.ac.uk/spm/software/ spm5/; Friston et al., 1998; Glover, 1999; Worsley et al., 2002) and fit the model to the averaged time course of the BOLD signal change for each condition and for each subject using the weighted least-square method.

\section{Behavioral experiment for checking attention control}

Although the effect of top-down attention was minimized by the attention control task, we conducted a behavioral experiment to further confirm that top-down attention did not differ across stimulus conditions.

Ten healthy adults participated (three of whom had also participated in the fMRI experiment; three females; mean age, 28.3 years). In this experiment, subjects were asked to do the same attention task (i.e., to count the appearance of a blue fixation point) as used in the fMRI experiment. However, each blocked session contained only one stimulus condition (e.g., always Mid Opposite in a certain run) to examine how much behavioral performance depended on stimulus condition. We presented images on a CRT monitor $(1600 \times 1200$ pixels; refresh rate, $60 \mathrm{~Hz}$; RDF223H; Mitsubishi Electric). Stimulus size, position, and eccentricity were matched to those in the scanner. Stimuli were viewed under dim illumination in a dark room. The viewing distance of $66 \mathrm{~cm}$ was maintained using a chin rest.

Each subject executed two runs for each of the seven stimulus conditions. In the analysis, we excluded outlier data that fell beyond 2 SDs from the mean, but the results did not change if they were included.

\section{Offline eye-movement recording}

Although the attention control task at the fixation point minimized voluntary eye movements, it remained possible that faster central stimuli triggered larger involuntary fixational eye movements, which could have resulted in greater BOLD responses.

Therefore, we conducted offline eye-movement recording for the same set of stimuli. Nine healthy adults (three of whom had also participated in the fMRI experiment; two females; mean age, 28.6 years) participated. The stimulus presentation methods were the same as those used in the behavioral experiment. We used an eye tracker (Eyelink; SR Research) to track horizontal and vertical movements of both eyes concurrently at $500 \mathrm{~Hz}$ for eight subjects and at $250 \mathrm{~Hz}$ for one subject.

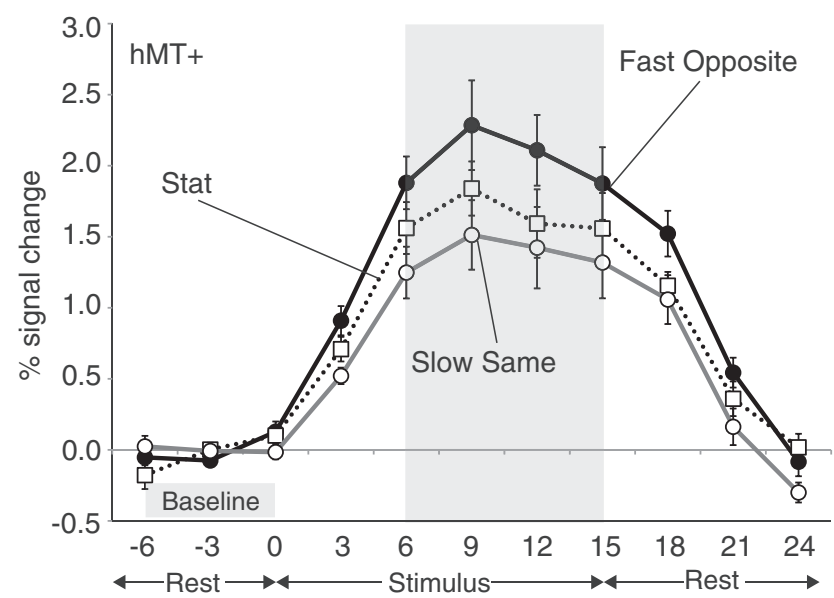

Time (s)

Figure 4. BOLD signal changes in hMT + averaged across nine subjects under three representative velocities (Fast Opposite, Stat, and Slow Same). Time 0 and time 15 indicate the stimulus onset and offset, respectively. Error bars indicate \pm 1 SEM.

Analysis of eye-movement data followed the method of Murakami (2004) (Murakami, 2004, 2010; Murakami et al., 2006; Ashida et al., 2012). Drift eye movements during fixation were analyzed along vertical and horizontal axes separately. Instantaneous drift velocities were computed by differentiating eye position data with the three-point differentiation algorithm by excluding those exceeding $10 \%$ as putative microsaccades (Bair and O'Keefe, 1998) and by low-pass filtering $(\sim 30$ $\mathrm{Hz}$ ) the velocity within the stimulus presentation. A histogram of instantaneous velocities was plotted with a bin width of $0.1 \%$. A Gaussian distribution was fitted by the least-square method, and its SD was taken as an index of fixational instability originating from eye drift.

The sequence of visual stimulus presentation was the same as in the main experiment. Two runs were conducted for each participant.

\section{Results}

\section{hMT + activity exhibits a pattern compatible with induced motion perception}

Figure 4 shows an example of the BOLD time course in response to visual stimulation. The data shown in this particular plot are derived from voxels that were within the intersection of the region activated by hMT + localizer and the region activated by the stimulus localizer. The data at three representative velocities are shown for illustrative purposes. The vertical axis indicates the BOLD signal change compared with the baseline signal averaged across the three scans taken before the stimulus onset $(-6,-3$, and $0 \mathrm{~s})$. For the subsequent analysis, we averaged the signal change values of four scans $(6,9,12$, and $15 \mathrm{~s}$ ) around the peak of the curve showing activation in response to the stimulus presentation.

Figure 5 shows the averaged signal changes in hMT + plotted across conditions. hMT + exhibited minimal activation under the Slow Same condition, in which the central stimulus was actually moving at the cancellation velocity for induced motion rather than under the Stat condition, in which the central stimulus was physically stationary. Under the Slow Same condition, the subjects perceived the central stimulus to be stationary because the physical motion and illusory induced motion perceptually canceled each other out. Activation under this condition was significantly less than that under the Fast Opposite, Slow Opposite, Stat, and Mid Same conditions (paired $t$ test using the Holm-Bonferroni correction; $p<0.005,0.01,0.05$, and 0.01 , respectively). The significant difference between the Slow Same and Stat conditions strongly supports the notion that hMT+ 


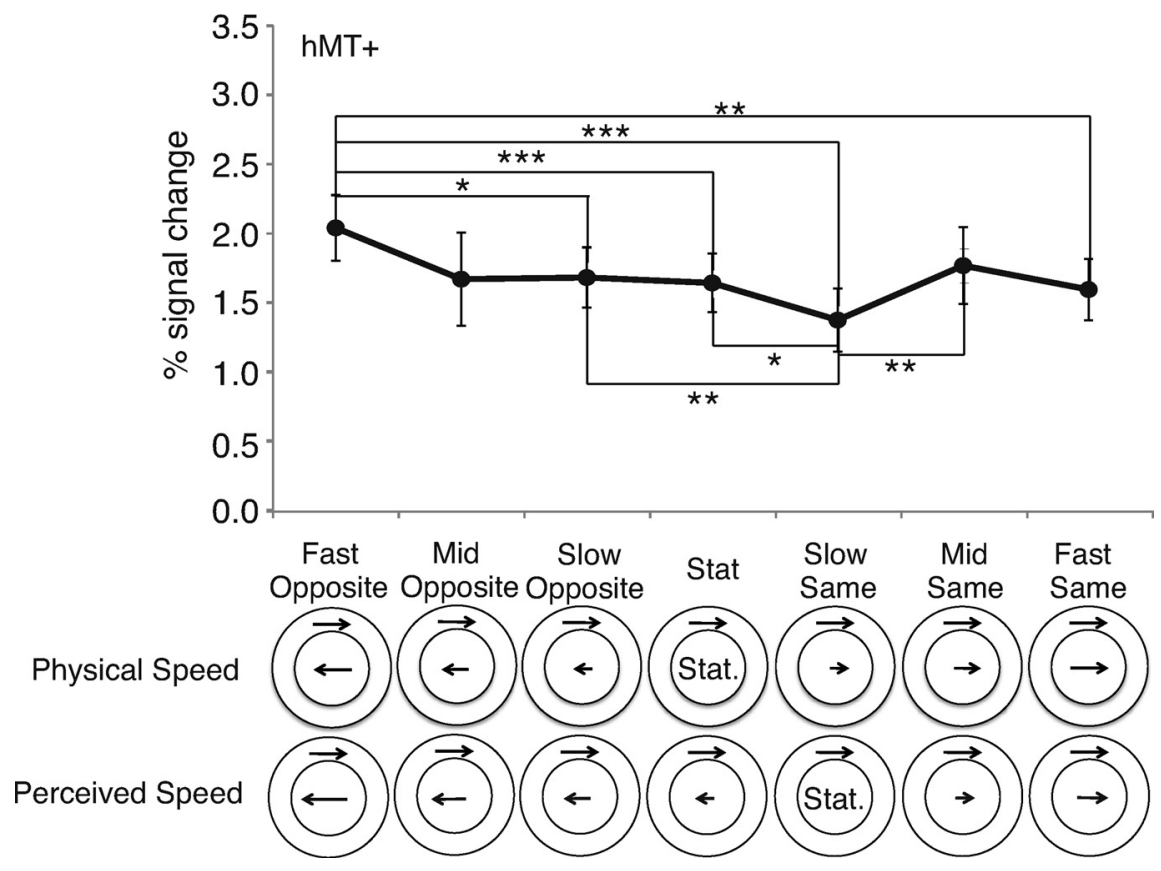

Figure 5. Signal changes in hMT + under all conditions plotted against the seven velocities. ${ }^{*} p<0.05,{ }^{* *} p<0.01$, and ${ }^{* * *} p<0.005$, significance levels of the differences compared with the Fast Opposite and Slow Same conditions. Error bars indicate \pm 1 SEM.

activation is minimal when the central stimulus is perceptually, but not physically, stationary. The significant difference between the Slow Same and Mid Same conditions also supported the idea that activation of the hMT + is compatible with perceived speed rather than the kinetic boundary. Furthermore, hMT + exhibited maximum activation under the Fast Opposite condition in which the central and surrounding stimuli moved in opposite directions at the same speed $(1 \% \mathrm{~s})$. Activation under this condition was significantly greater than that under the Slow Opposite, Stat, Slow Same, and Fast Same conditions (paired $t$ test using the Holm-Bonferroni correction; $p<0.05,0.005,0.005$, and 0.01 , respectively). The significantly greater hMT + activation under the Fast Opposite compared with the Fast Same condition suggests that hMT + activation was modulated by the direction of the surrounding stimulus, although the physical speeds of both the central and surrounding stimuli were equivalent under these two conditions. This result agrees with previous studies showing that hMT + exhibited significant activation in response to opposing motions (Shulman et al., 1998; Moutsiana et al., 2011). Overall, these results support the hypothesis that visual responses in $\mathrm{hMT}+$ are related to the perceived speed (Fig. $1 \mathrm{~A}$, red line) rather than the physical speed of the central stimulus or the kinetic boundary between the central and surrounding stimulus.

We observed high activation in hMT + even when the central stimulus was physically or perceptually stationary (Fig. $5 ;>1 \%$ signal change). These high activations are not surprising because a fraction of such responses naturally originated from the surrounding stimulus. Particularly in hMT + , the large pRF size made it impossible to isolate "center-only" voxels. However, this effect did not change the interpretation of data in hMT + because of the following reasons. First, we used the same surroundstimulus speed across the seven stimulus conditions (Fig. 1B). Thus, the main effect of the surrounding stimulus itself was removed. Second, the pattern of activation in hMT + remained unchanged when we changed the criterion of significant voxels, as we will describe below (see No effect of the stimulus localizer threshold).

\section{No obvious difference in activation between two retinotopy-based maps within hMT +} These results were consistent between the anterior and posterior regions of hMT + . Studies in monkeys have reported directionally-specific surround modulation in MT and MSTl (ventral lateral MST) neurons (Allman et al., 1985; Tanaka et al., 1986; Eifuku and Wurtz, 1998; Born et al., 2000; Perge et al., 2005). In humans, hMT + includes two retinotopic maps (TO-1 and TO-2), the boundary of which corresponds to the boundary between the two functionally defined areas, hMT and hMST (Dukelow et al., 2001; Huk et al., 2002; Amano et al., 2009b). We divided hMT + into TO- 1 and TO-2 based on retinotopy. Figure $6 \mathrm{~A}$ shows an example of retinotopic maps for a representative subject. We found a clear reversal of retinotopy (representation of upper vertical meridian) within hMT + in 13 of 18 hemispheres, whereas the remaining five hemispheres showed intermixed patterns. By using the pRF estimation method used in previous studies (Dumoulin and Wandell, 2008; Amano et al., $2009 \mathrm{~b}$ ), we also confirmed that the pRF sizes in TO-2 were larger than those in TO-1 (Fig. $6 \mathrm{~B}$ ) in a qualitatively consistent manner with previous studies (Amano et al., 2009b; Winawer et al., 2010).

The activity patterns were almost identical between TO- 1 and TO-2 (Fig. 6C); both activities were related to perceived speed rather than to physical speed. Even if we excluded the data with intermixed retinotopy maps, we did not find any difference between TO-1 and TO-2.

\section{Comparison with early visual areas}

The differences across conditions were generally smaller than those observed in hMT + in the other visual areas $(\mathrm{V} 1, \mathrm{~V} 2, \mathrm{~V} 3$, and V3A). In these areas, the pattern of activation was similar to that of hMT + , with the highest level of activation observed under the Fast Opposite condition and the lowest observed under the Slow Same condition, but statistically significant differences were found in only a few cases. In V1, no significant difference was observed across conditions. In V2, activation under the Slow Same condition was less than that under the Mid Same condition (paired $t$ test using the Holm-Bonferroni correction; $p<0.05$ ). In V3, activation under the Fast Opposite condition was greater than that under the Slow Opposite and Slow Same conditions (paired $t$ test using the Holm-Bonferroni correction; $p<0.05$ ), and activation under the Slow Same condition was less than that under the Fast Opposite and Mid Same conditions (paired $t$ test using the Holm-Bonferroni correction; $p<0.05$ ). In V3A, activation under the Fast Opposite condition was greater than that under the Slow Same condition (paired $t$ test using the HolmBonferroni correction; $p<0.005)$, Mid Opposite $(p<0.05)$, and Slow Opposite conditions $(p<0.05)$, and activation under the Slow Same condition was less than that under the Fast Opposite (paired $t$ test using the Holm-Bonferroni correction; $p<0.005$ ) 
and Stat $(p<0.05)$ conditions. This statistical pattern did not improve when the threshold for ROI determination was changed.

The visual responses were compared across areas by normalizing the signal changes of each area relative to the signal change under the Fast Opposite condition (Fig. 7A). hMT + showed the greatest variation in normalized visual responses across conditions. To examine the correspondence between activation in each area and perceived speed, we calculated the perceived speed index as the difference in visual responses between the two conditions in which the central stimulus should appear fastest and slowest, namely the Fast Opposite and Slow Same conditions: Perceived Speed Index $=($ Fast Opposite - Slow Same)/(Fast Opposite + Slow Same).

Figure $7 B$ shows the perceived speed index in each area. We found a significant difference in the perceived speed index between areas (Kruskal-Wallis test, $H_{(4)}$ $=11.90 ; p=0.018)$. The post hoc analysis revealed that the perceived speed index in hMT+ was greater than that in V1 (Scheffé's test, $p<0.05$ ) and that no significant difference was found among the other areas $(\mathrm{V} 1-\mathrm{V} 3 \mathrm{~A})$.

\section{$\mathrm{hMT}+$ shows a robust pattern of activation regardless of the response amplitude definition}

We defined the response amplitude by averaging the signal change values of four scans $(6,9,12$, and $15 \mathrm{~s}$ after stimulus onset) around the peak of the curve showing activation in response to the stimulus presentation. To determine whether the present finding depended on this particular definition of response amplitude, we redefined the response amplitude by using model responses (the stimulus time course convolved with the HRF; see Materials and Methods). In hMT+, the statistical significance was almost unchanged such that the activation under the Slow Same condition was significantly less than that under the Fast Opposite, Slow Opposite, Stat, and Mid Same conditions (paired $t$ test using the Holm-Bonferroni correction; $p<0.005,0.01,0.05$, and 0.05 , respectively), whereas activation under the Fast Opposite condition was significantly greater than that under the Slow Opposite, Stat, Slow Same, and Fast Same conditions (paired $t$ test using the Holm-Bonferroni correction; $p<0.005,0.01,0.005$, and 0.05 , respectively). In contrast, the statistical significance pattern in the early visual areas was changed slightly from the original analysis. In V1, the difference between Fast Opposite and Slow Same and that between Stat and Slow Same became significant (paired $t$ test using the Holm-Bonferroni correction; $p<0.05$ and 0.05 , respectively). In contrast, no significant difference was observed across conditions in V2. Activation under the Fast Opposite condition was greater than that under the Slow Same condition in V3 and V3A (paired $t$ test using the Holm-Bonferroni correction; $p<$
0.05), but other significant differences observed in the original analysis became nonsignificant. These results suggest that differences in activation across conditions in hMT + were robust and independent of the analysis method, whereas those observed in early visual areas (V1-V3A) were unstable.

\section{No effect of the stimulus localizer threshold}

To confirm the robustness of the data, we manipulated the stimulus localizer threshold. In hMT + , a large enough number of voxels showing significant responses to the stimulus localizer remained significant in five subjects when we used a stricter threshold for the significance criterion (uncorrected $p<0.005$ ). Figure $7 C$ shows the normalized signal change in each area with the stricter threshold. It is evident that hMT + activation exhibited a response pattern that was most compatible with perceived speed compared with data in the early visual areas (V1-V3A), which became noisier than the original data. As we applied a much stricter threshold (e.g., $q<0.05$, FDR corrected), the response patterns in the early visual areas became even noisier and less compatible with any model, let alone the perceived speed hypothesis (data not shown). Thus, the present finding of compatibility 

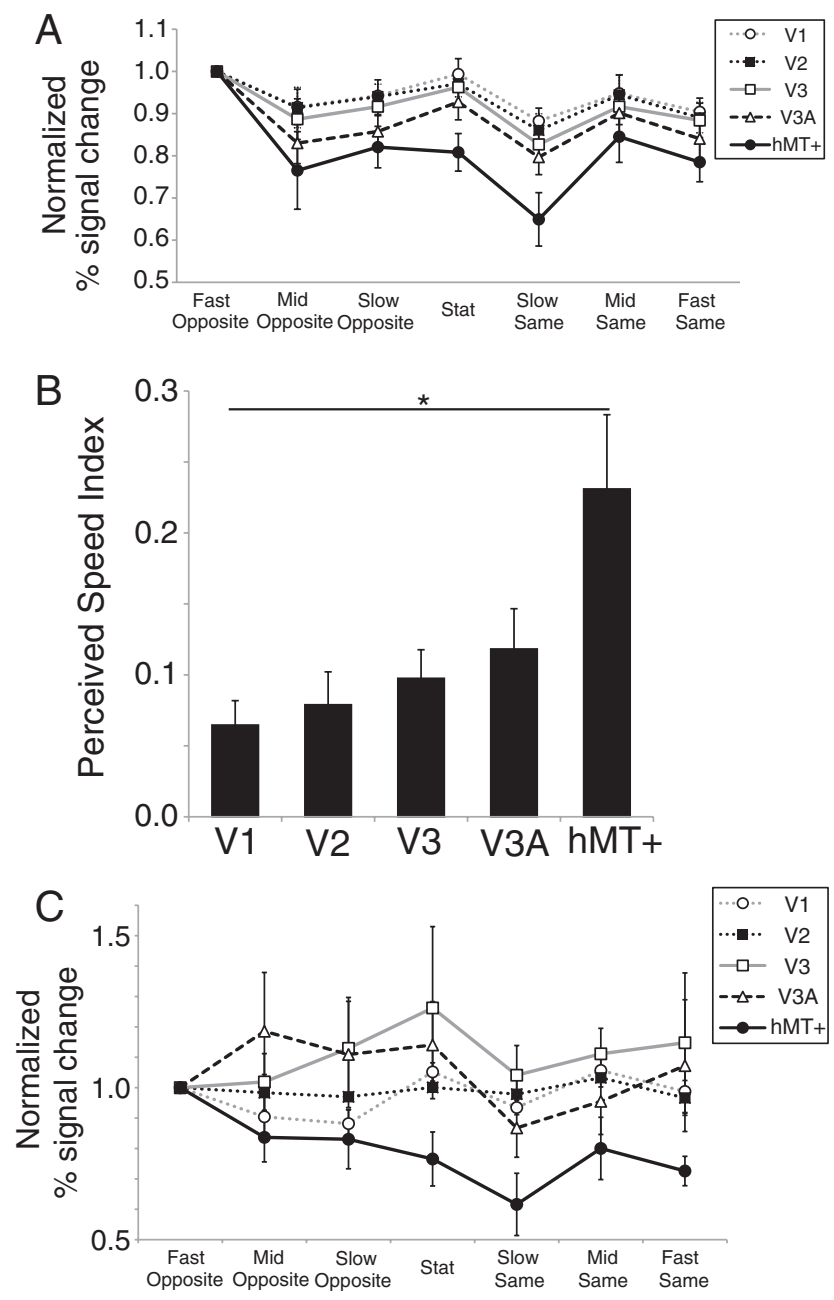

Figure 7. A, Normalized activity in different cortical areas. The BOLD signal change in each condition was divided by that observed under the Fast Opposite condition. Different curves indicate different areas (see inset). Error bars indicate \pm 1 SEM. $\boldsymbol{B}$, Perceived speed index. The horizontal axis represents areas (V1, V2, V3, V3A, and hMT + ), and the vertical axis represents the perceived speed index in each area. ${ }^{*} p<0.05$, statistical difference between areas by Scheffé's test. Error bars indicate \pm 1 SEM. C, Normalized activity in different ROls with stricter stimulus localizer cutoffs ( $p<0.005$, uncorrected).

between hMT + activity and the perception of induced motion was independent of the method of selecting significant voxels.

\section{Stable attention control across stimulus conditions}

We conducted a behavioral experiment to confirm that control of top-down attention did not differ across stimulus conditions ( $n=10$; see Materials and Methods). Average \pm SD accuracy rates under the seven stimulus conditions (in the order presented in Fig. $1 B$ ) were $0.99 \pm 0.009,0.982 \pm 0.028,0.987 \pm 0.014$, $0.99 \pm 0.01,0.984 \pm 0.011,0.988 \pm 0.009$, and $0.978 \pm 0.019$. No significant main effect of stimulus condition was found (one-way ANOVA on the accuracy of performance indicating the degree of attention control, $\left.F_{(6,63)}=0.88, p=0.52\right)$. This refutes the idea that top-down attention might have caused the differences in BOLD signals across conditions obtained in our fMRI experiment.

\section{Statistics of fixational eye movements}

We also conducted an offline recording of fixational eye movements $(n=9)$ during stimulus presentation under each stimulus

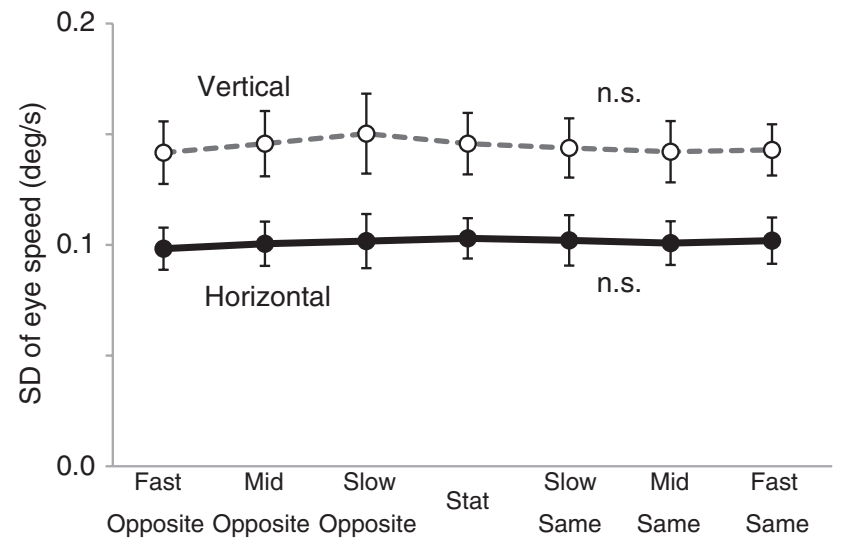

Figure 8. Results of eye-movement recording $(n=9)$. Vertical axis represents the variability in eye speeds (SD of the Gaussian fitted to the histogram of instantaneous velocities during fixation). The black solid curve with black circles indicates horizontal speed, and the gray dotted curve with white circles indicates vertical speed. Error bars indicate \pm 1 SEM.

condition. The fixational instability, as quantified by the SD of instantaneous ocular drift velocities during fixation (see Materials and Methods), is plotted for each of the seven stimulus conditions (Fig. 8). We found no significant main effect of stimulus condition in the one-way $\operatorname{ANOVA}\left(F_{(6,56)}=0.02, p>0.99\right.$ for horizontal eye velocity; $F_{(6,56)}=0.04, p>0.99$ for vertical eye velocity). This indicates no measurable difference in the amplitude of any slow oculomotor control, including fixational drift, pursuit, ocular following response, and slow phases of optokinetic nystagmus across stimulus conditions. We also analyzed the frequency of blinks and microsaccades, but we found no significant main effect of stimulus condition (one-way ANOVA; $F_{(6,56)}=$ $0.14, p=0.99$ for blinks; $F_{(6,56)}=0.37, p=0.9$ for microsaccades). Thus, it is highly unlikely that a difference in eye movements was the cause of the observed differences in BOLD signals across conditions in our fMRI experiment.

\section{Discussion}

Relationship between cortical activity and induced motion

The present study used fMRI to reveal the relationship between induced motion perception and cortical activation in the human brain. We found that hMT + activation increased when the central and surrounding stimuli moved in directions opposite to each other and was the lowest when the central stimulus appeared stationary at the point of perceptual cancellation between physical and induced motions. Furthermore, the patterns of activity exhibited in hMT + were more compatible with perceived speed than those observed in other areas such as V1. These results suggest that hMT+ activation is a neural correlate of induced motion perception rather than of physical speed or kinetic boundary characterized by relative motion.

Induced motion has been classically interpreted in terms of lateral inhibition among motion detectors, called surround suppression in more contemporary terminology (Walker and Powell, 1974; Tynan and Sekuler, 1975). Murakami and Shimojo $(1993,1996)$ demonstrated that the optimal stimulus size to elicit this illusion changes with eccentricity, which is analogous to the finding that the classical receptive-field size of MT neurons increases in proportion to eccentricity. Our results showing a relationship between hMT + activation and induced motion agree with the findings of these previous psychophysical studies. Furthermore, the present results agree with recent computational models demonstrating a possible relationship between popula- 
tion activities in area MT neurons and induced motion (Tzvetanov and Womelsdorf, 2008; Tajima et al., 2010b).

Our results have two primary implications. First, they provide new evidence of contextual modulation in the human brain. Although surround suppression at the single-neuron level is well established (Blakemore and Tobin, 1972; Allman et al., 1985; Tanaka et al., 1986; Knierim and van Essen, 1992; Eifuku and Wurtz, 1998), it is not clear how such contextual modulation is organized in a large-scale neural network. Previous fMRI studies have reported suppression of cortical activation in the presence of surrounding stimuli (Kastner et al., 1998, 2001; Williams et al., 2003; Zenger-Landolt and Heeger, 2003; McDonald et al., 2009; Tajima et al., 2010a; Zuiderbaan et al., 2012). However, our study is the first to demonstrate contextual modulation of large-scale activity in motion-related areas in a manner qualitatively similar to surround suppression at a single-neuron level (Allman et al., 1985; Tanaka et al., 1986; Eifuku and Wurtz, 1998) in that the center and surround moving in the same direction yield lower activation.

Second, the present results dissociate motion contrast from the kinetic boundary. Previous studies have not clarified which of these two factors in hMT + activation is more important because a comparison has only been made between unidirectional motion and opposing motions (Shulman et al., 1998; Moutsiana et al., 2011). In the present study, we systematically manipulated the velocity of the central stimulus and found that hMT + activation was more compatible with induced motion than with the presence/absence of a difference in physical speed. The present results constitute the first demonstration of the neural activation pattern related to subjective perception of induced motion distinct from the neural responses to motion-defined boundaries found in widespread areas (Lamme et al., 1993; Dupont et al., 1997; Reppas et al., 1997; van Oostende et al., 1997; Zeki et al., 2003; Larsson and Heeger, 2006, 2010). The present results suggest that $\mathrm{hMT}+$ is involved in a mechanism that contrasts motion signals between relatively large portions of the visual field and that can generate a strong perceptual bias in motion perception if no other reliable visual cue is available, as in our experimental display.

\section{Distinction between hMT and hMST}

The characteristics of surround suppression slightly differ between the macaque MT and MSTl (Tanaka et al., 1986; Eifuku and Wurtz, 1998). In MT, neurons are not activated when a stimulus inside the classical receptive field is stationary, even when the surround is moving (Tanaka et al., 1986). In contrast, MSTl neurons are activated in such a case (Eifuku and Wurtz, 1998). In the present study, more MSTl-like activities were observed in hMT + , which showed a higher level of activation under the Stat than under the Slow Same condition (Fig. 5). In light of this disparity, we compared activation patterns between the two subdivisions of hMT+, TO-1 and TO-2 (Amano et al., 2009b), and confirmed virtually identical activity patterns. Two possible explanations are worth mentioning. First, interspecies differences may exist in MT and MST functions, as reported previously (Wall et al., 2008). Second, strong interconnections between the two areas may obscure a clear distinction between their activity patterns at the level of BOLD signals. Macroscopic activation may result in a different signature than the expected sum of individual neuronal activities (Bartels et al., 2008).

\section{Speed representation in the cortex}

Most speed-selective neurons in the monkey MT and MST respond maximally to high speeds (e.g., 16\%; Maunsell and Van
Essen, 1983; Lagae et al., 1993; Cheng et al., 1994; Kawano et al., 1994; Duffy and Wurtz, 1997; Perrone and Thiele, 2001; Liu and Newsome, 2003; Priebe et al., 2003), and in humans, hMT+ exhibits large magnetoencephalography (MEG) responses at fast speeds (Kawakami et al., 2002; Amano et al., 2009a). However, the speeds used in the present study were substantially slower $(1 \%$ at maximum) than those associated with the tuning peaks of MT and MST neurons and were well within the range of the ascending slope of the speed function. Thus, hMT + responses depending on perceived speed, as shown in Figure 5, are consistent with the findings of previous studies of speed tuning.

A question remains as to the discontinuous pattern of activation in hMT + , which resembles a step function (Fig. 5) rather than a smooth increase with perceived speed. Two possible explanations may account for this finding. First, our results may have revealed a genuine pattern of activation for the range of slow speeds used (up to $1 \%$ ). MEG studies have shown gradual changes in activity using a speed stimulus (Kawakami et al., 2002; Amano et al., 2005, 2009a); however, the changes were observed only across a very large speed range (e.g., $0.4-500^{\circ} / \mathrm{s}$ ). Within the narrower and slower speed range used in the present study, the response magnitudes may have been able to distinguish only the three perceptual states of "stationary," "barely noticeable motion," and "definite motion." The second possibility is that the discrete pattern reflects response characteristics specific to BOLD signals. The speed tuning of BOLD signals is not well understood; however, recent fMRI studies showing speed selectivity in hMT+ using fMRI adaptation (Lingnau et al., 2009) and multi-voxel pattern analysis (Vintch and Gardner, 2011) indicate that the BOLD signals in hMT + represent speed in a highly nonlinear and implicit manner. Future brain-imaging studies are needed to clarify the complex nature of speed representation in hMT + .

\section{Activity in other visual areas}

In areas $\mathrm{V} 1, \mathrm{~V} 2, \mathrm{~V} 3$, and $\mathrm{V} 3 \mathrm{~A}$, the differences in activation across conditions were not statistically significant in most cases, and the pattern of significance changed depending on the method of analysis. Three possible explanations for this minor correlation with perceived speed are as follows. (1) The stimulus size and eccentricity were optimized to elicit a sufficiently strong induced motion (Murakami and Shimojo, 1993, 1996), and, as a result, they may have been suboptimal for neurons in these visual areas. However, our central stimulus, sized $2^{\circ}$ at $5.33^{\circ}$ eccentricity, fell within the receptive-field size variability in extrastriate areas (Albright and Desimone, 1987), and neurons with small receptive fields could code differential motion if they straddled the central and surrounding stimuli. (2) These areas may contain perceivedspeed-selective neurons in smaller proportion than hMT + does. Although the monkey area V1 contains neurons showing direction-dependent surround suppression (Jones et al., 2001), the proportion of direction-selective neurons is generally smaller than that found in area MT (Hawken et al., 1988), and the difference in the proportion of neurons might affect the results, as argued in a recent report (Lee and Lee, 2012). However, neuroimaging studies have also demonstrated a greater directionselective responses in V3A (Nishida et al., 2003; Ashida et al., 2007) or even in V1 (Huk et al., 2001; Kamitani and Tong, 2006; Ales and Norcia, 2009); hence; limited cellular proportion may not limit the BOLD signal change. (3) The observed pattern of activation in the early visual areas may reflect feedback signals from higher-order visual areas, such as hMT + . It is difficult to deconstruct signals into feedforward and feedback components in fMRI or to clarify whether the higher compatibility between 
$\mathrm{hMT}+$ activation and induced motion is generated within hMT + or whether it is inherited from a subset of neurons in early visual areas. However, in light of more robust activation in $\mathrm{hMT}+$, parsimony might suggest that the activation pattern in $\mathrm{hMT}+$ originates from this area and is transferred backward to early visual areas through feedback signals. Future investigations with various research techniques (e.g., MEG) may provide an answer to this question.

\section{References}

Albright TD, Desimone R (1987) Local precision of visuotopic organization in the middle temporal area (MT) of the macaque. Exp Brain Res 65:582-592.

Ales JM, Norcia AM (2009) Assessing direction-specific adaptation using the steady-state visual evoked potential: results from EEG source imaging. J Vis 9(7):8 1-13.

Allman J, Miezin F, McGuinness E (1985) Direction- and velocity-specific responses from beyond the classical receptive field in the middle temporal visual area (MT). Perception 14:105-126.

Amano K, Kuriki I, Takeda T (2005) Direction-specific adaptation of magnetic responses to motion onset. Vision Res 45:2533-2548.

Amano K, Kimura T, Nishida S, Takeda T, Gomi H (2009a) Close similarity between spatiotemporal frequency tunings of human cortical responses and involuntary manual following responses to visual motion. J Neurophysiol 101:888-897.

Amano K, Wandell BA, Dumoulin SO (2009b) Visual field maps, population receptive field sizes, and visual field coverage in the human MT+ complex. J Neurophysiol 102:2704-2718.

Ashida H, Lingnau A, Wall MB, Smith AT (2007) fMRI adaptation reveals separate mechanisms for first-order and second-order motion. J Neurophysiol 97:1319-1325.

Ashida H, Kuriki I, Murakami I, Hisakata R, Kitaoka A (2012) Directionspecific fMRI adaptation reveals the visual cortical network underlying "Rotating Snakes" illusion. Neuroimage 61:1143-1152.

Bair W, O'Keefe LP (1998) The influence of fixational eye movements on the response of neurons in area MT of the macaque. Vis Neurosci 15:779-786.

Baker CL Jr, Braddick OJ (1982) Does segregation of differently moving areas depend on relative or absolute displacement? Vision Res 22:851-856.

Bartels A, Logothetis NK, Moutoussis K (2008) fMRI and its interpretations: an illustration on directional selectivity in area V5/MT. Trends Neurosci 31:444-453.

Blakemore C, Tobin EA (1972) Lateral inhibition between orientation detectors in the cat's visual cortex. Exp Brain Res 15:439-440.

Born RT, Groh JM, Zhao R, Lukasewycz SJ (2000) Segregation of object and background motion in visual area MT: effects of microstimulation on eye movements. Neuron 26:725-734

Brainard DH (1997) The Psychophysics Toolbox. Spat Vis 10:433-436.

Cheng K, Hasegawa T, Saleem KS, Tanaka K (1994) Comparison of neuronal selectivity for stimulus speed, length, and contrast in the prestriate visual areas V4 and MT of the macaque monkey. J Neurophysiol 71:2269-2280.

Duffy CJ, Wurtz RH (1997) Medial superior temporal area neurons respond to speed patterns in optic flow. J Neurosci 17:2839-2851.

Dukelow SP, DeSouza JF, Culham JC, van den Berg AV, Menon RS, Vilis T (2001) Distinguishing subregions of the human MT plus complex using visual fields and pursuit eye movements. J Neurophysiol 86:1991-2000.

Dumoulin SO, Wandell BA (2008) Population receptive field estimates in human visual cortex. Neuroimage 39:647-660.

Dumoulin SO, Bittar RG, Kabani NJ, Baker CL Jr, Le Goualher G, Bruce Pike G, Evans AC (2000) A new anatomical landmark for reliable identification of human area V5/MT: a quantitative analysis of sulcal patterning. Cereb Cortex 10:454-463.

Duncker L (1929) Über induzierte Bewegung, Psychologische Forschung 12:180-259. In: Source book of Gestalt psychology (1950) (Ellis WD, ed and translator), pp 161-172. London: Kegan Paul, Trench, Trübner and Co.

Dupont P, De Bruyn B, Vandenberghe R, Rosier AM, Michiels J, Marchal G, Mortelmans L, Orban GA (1997) The kinetic occipital region in human visual cortex. Cereb Cortex 7:283-292.
Eifuku S, Wurtz RH (1998) Response to motion in extrastriate area MSTl: center-surround interactions. J Neurophysiol 80:282-296.

Engel SA, Glover GH, Wandell BA (1997) Retinotopic organization in human visual cortex and the spatial precision of functional MRI. Cereb Cortex 7:181-192.

Friston KJ, Fletcher P, Josephs O, Holmes A, Rugg MD, Turner R (1998) Event-related fMRI: characterizing differential responses. Neuroimage 7:30-40.

Furmanski CS, Engel SA (2000) An oblique effect in human primary visual cortex. Nat Neurosci 3:535-536.

Glover GH (1999) Deconvolution of impulse response in event-related BOLD fMRI. Neuroimage 9:416-429.

Harvey BM, Dumoulin SO (2011) The relationship between cortical magnification factor and population receptive field size in human visual cortex: constancies in cortical architecture. J Neurosci 31:13604-13612.

Hawken MJ, Parker AJ, Lund JS (1988) Laminar organization and contrast sensitivity of direction-selective cells in the striate cortex of the Old World monkey. J Neurosci 8:3541-3548.

Huk AC, Ress D, Heeger DJ (2001) Neuronal basis of motion aftereffect reconsidered. Neuron 32:161-172.

Huk AC, Dougherty RF, Heeger DJ (2002) Retinotopy and functional subdivision of human areas MT and MST. J Neurosci 22:7195-7205.

Jones HE, Grieve KL, Wang W, Sillito AM (2001) Surround suppression in primate V1. J Neurophysiol 86:2011-2028.

Kamitani Y, Tong F (2006) Decoding seen and attended motion directions from activity in the human visual cortex. Curr Biol 16:1096-1102.

Kastner S, De Weerd P, Desimone R, Ungerleider LG (1998) Mechanisms of directed attention in the human extrastriate cortex as revealed by functional MRI. Science 282:108-111.

Kastner S, De Weerd P, Pinsk MA, Elizondo MI, Desimone R, Ungerleider LG (2001) Modulation of sensory suppression: implications for receptive field sizes in the human visual cortex. J Neurophysiol 86:1398-1411.

Kawakami O, Kaneoke Y, Maruyama K, Kakigi R, Okada T, Sadato N, Yonekura Y (2002) Visual detection of motion speed in humans: spatiotemporal analysis by fMRI and MEG. Hum Brain Mapp 16:104-118.

Kawano K, Shidara M, Watanabe Y, Yamane S (1994) Neural activity in cortical area MST of alert monkey during ocular following responses. J Neurophysiol 71:2305-2324.

Knierim JJ, van Essen DC (1992) Neuronal responses to static texture patterns in area V1 of the alert macaque monkey. J Neurophysiol 67:961-980.

Kuriki I, Ashida H, Murakami I, Kitaoka A (2008) Functional brain imaging of the Rotating Snakes illusion by fMRI. J Vis 8(10):16 1-10.

Lagae L, Raiguel S, Orban GA (1993) Speed and direction selectivity of macaque middle temporal neurons. J Neurophysiol 69:19-39.

Lamme VA, van Dijk BW, Spekreijse H (1993) Contour from motion processing occurs in primary visual cortex. Nature 363:541-543.

Larsson J, Heeger DJ (2006) Two retinotopic areas in human lateral occipital cortex. J Neurosci 26:13128-13142.

Larsson J, Heeger DJ, Landy MS (2010) Orientation selectivity of motionboundary responses in human visual cortex. J Neurophysiol 104:2940-2950

Lee HA, Lee SH (2012) Hierarchy of direction-tuned motion adaptation in human visual cortex. J Neurophysiol 107:2163-2184.

Lingnau A, Ashida H, Wall MB, Smith AT (2009) Speed encoding in human visual cortex revealed by fMRI adaptation. J Vis 9(13):3 1-15.

Liu J, Newsome WT (2003) Functional organization of speed tuned neurons in visual area MT. J Neurophysiol 89:246-256.

Maunsell JH, Van Essen DC (1983) Functional properties of neurons in middle temporal visual area of the macaque monkey. I. Selectivity for stimulus direction, speed, and orientation. J Neurophysiol 49:1127-1147.

McDonald JS, Seymour KJ, Schira MM, Spehar B, Clifford CW (2009) Orientation-specific contextual modulation of the fMRI BOLD response to luminance and chromatic gratings in human visual cortex. Vision Res 49:1397-1405.

Moutsiana C, Field DT, Harris JP (2011) The neural basis of center-surround interaction in visual motion processing. PLoS One 6:e22902.

Murakami I (2004) Correlations between fixation stability and visual motion sensitivity. Vision Res 44:751-761.

Murakami I (2010) Eye movements during fixation as velocity noise in minimum-motion detection. Jpn Psychol Res 52:54-66.

Murakami I, Shimojo S (1993) Motion capture changes to induced motion 
at higher luminance contrasts, smaller eccentricities, and larger inducer sizes. Vision Res 33:2091-2107.

Murakami I, Shimojo S (1996) Assimilation-type and contrast-type bias of motion induced by the surround in a random-dot display: evidence for center-surround antagonism. Vision Res 36:3629-3639.

Murakami I, Kitaoka A, Ashida H (2006) A positive correlation between fixation instability and the strength of illusory motion in a static display. Vision Res 46:2421-2431.

Nishida S, Sasaki Y, Murakami I, Watanabe T, Tootell RB (2003) Neuroimaging of direction-selective mechanisms for second-order motion. J Neurophysiol 90:3242-3254.

Perge JA, Borghuis BG, Bours RJ, Lankheet MJ, van Wezel RJ (2005) Dynamics of directional selectivity in MT receptive field centre and surround. Eur J Neurosci 22:2049-2058.

Perrone JA, Thiele A (2001) Speed skills: measuring the visual speed analyzing properties of primate MT neurons. Nat Neurosci 4:526-532.

Pitzalis S, Sereno MI, Committeri G, Fattori P, Galati G, Patria F, Galletti C (2010) Human V6: the medial motion area. Cereb Cortex 20:411-424.

Priebe NJ, Cassanello CR, Lisberger SG (2003) The neural representation of speed in macaque area MT/V5. J Neurosci 23:5650-5661.

Regan D (1989) Orientation discrimination for objects defined by relative motion and objects defined by luminance contrast. Vision Res 29:1389-1400.

Reinhardt-Rutland AH (1988) Induced movement in the visual modality: an overview. Psychol Bull 103:57-71.

Reppas JB, Niyogi S, Dale AM, Sereno MI, Tootell RB (1997) Representation of motion boundaries in retinotopic human visual cortical areas. Nature 388:175-179.

Sereno MI, McDonald CT, Allman JM (1994) Analysis of retinotopic maps in extrastriate cortex. Cereb Cortex 4:601-620.

Sereno MI, Dale AM, Reppas JB, Kwong KK, Belliveau JW, Brady TJ, Rosen BR, Tootell RB (1995) Borders of multiple visual areas in humans revealed by functional magnetic resonance imaging. Science 268:889-893.

Shulman GL, Schwarz J, Miezin FM, Petersen SE (1998) Effect of motion contrast on human cortical responses to moving stimuli. J Neurophysiol 79:2794-2803.

Tadin D, Lappin JS, Gilroy LA, Blake R (2003) Perceptual consequences of centre-surround antagonism in visual motion processing. Nature 424:312-315

Tajima S, Watanabe M, Imai C, Ueno K, Asamizuya T, Sun P, Tanaka K, Cheng K (2010a) Opposing effects of contextual surround in human early visual cortex revealed by functional magnetic resonance imaging with continuously modulated visual stimuli. J Neurosci 30:3264-3270.

Tajima S, Takemura H, Murakami I, Okada M (2010b) Neuronal population decoding explains the change in signal detection sensitivity caused by task-irrelevant perceptual bias. Neural Comput 22:2586-2614.

Tanaka K, Hikosaka K, Saito H, Yukie M, Fukada Y, Iwai E (1986) Analysis of local and wide-field movements in the superior temporal visual areas of the macaque monkey. J Neurosci 6:134-144.
Tootell RB, Reppas JB, Kwong KK, Malach R, Born RT, Brady TJ, Rosen BR, Belliveau JW (1995) Functional analysis of human MT and related visual cortical areas using magnetic resonance imaging. J Neurosci 15:3215-3230.

Tynan P, Sekuler R (1975) Simultaneous motion contrast: velocity, sensitivity and depth response. Vision Res 15:1231-1238.

Tzvetanov T, Womelsdorf T (2008) Predicting human perceptual decisions by decoding neuronal information profiles. Biol Cybern 98:397-411.

Van Oostende S, Sunaert S, Van Hecke P, Marchal G, Orban GA (1997) The kinetic occipital (KO) region in man: an fMRI study. Cereb Cortex 7:690-701.

Vintch B, Gardner JL (2011) Decoding the Bayesian perception of speed in human visual cortex. Presented at the Eighth Annual Computational and Systems Neuroscience (CoSyNe) Meeting, Salt Lake City, UT, February 24-27 (slide session).

Walker P, Powell DJ (1974) Lateral interaction between neural channels sensitive to velocity in the human visual system. Nature 252:732-733.

Wall MB, Lingnau A, Ashida H, Smith AT (2008) Selective visual responses to expansion and rotation in the human MT complex revealed by functional magnetic resonance imaging adaptation. Eur J Neurosci 27:2747-2757.

Wandell BA, Winawer J (2011) Imaging retinotopic maps in the human brain. Vision Res 51:718-737.

Watson JD, Myers R, Frackowiak RS, Hajnal JV, Woods RP, Mazziotta JC, Shipp S, Zeki S (1993) Area V5 of the human brain: evidence from a combined study using positron emission tomography and magnetic resonance imaging. Cereb Cortex 3:79-94.

Wichmann FA, Hill NJ (2001a) The psychometric function. I. Fitting, sampling and goodness-of-fit. Percept Psychophys 63:1293-1313.

Wichmann FA, Hill NJ (2001b) The psychometric function. II. Bootstrapbased confidence intervals and sampling. Percept Psychophys 63:1314-1329.

Williams AL, Singh KD, Smith AT (2003) Surround modulation measured with functional MRI in the human visual cortex. J Neurophysiol 89:525-533.

Winawer J, Horiguchi H, Sayres RA, Amano K, Wandell BA (2010) Mapping hV4 and ventral occipital cortex: the venous eclipse. J Vis 10(5):1 $1-22$.

Worsley KJ, Liao CH, Aston J, Petre V, Duncan GH, Morales F, Evans AC (2002) A general statistical analysis for fMRI data. Neuroimage 15:1-15.

Zeki S, Watson JD, Lueck CJ, Friston KJ, Kennard C, Frackowiak RS (1991) A direct demonstration of functional specialization in human visual cortex. J Neurosci 11:641-649.

Zeki S, Perry RJ, Bartels A (2003) The processing of kinetic contours in the brain. Cereb Cortex 13:189-202.

Zenger-Landolt B, Heeger DJ (2003) Response suppression in V1 agrees with psychophysics of surround masking. J Neurosci 23:6884-6893.

Zuiderbaan W, Harvey BM, Dumoulin SO (2012) Modeling center-surround configurations in population receptive fields using fMRI. J Vis 12(3):10 1-15. 